\title{
Correction to: Calycosin inhibits the in vitro and in vivo growth of breast cancer cells through WDR7-7-GPR30 Signaling
}

Jing Tian ${ }^{1}$, Yong Wang ${ }^{2}$, Xing Zhang ${ }^{1}$, Qianyao Ren ${ }^{1}$, Rong Li ${ }^{1}$, Yue Huang ${ }^{3}$, Huiling Lu ${ }^{4}$ and Jian Chen ${ }^{*}$

\section{Correction}

In the publication of this article [1], the molecule weight of GPR30 in figures was incorrectly, this should have been $55 \mathrm{kDa}$, and not $38 \mathrm{kDa}$.

This has now been included in this erratum.

\begin{abstract}
Author details
'Key Laboratory of Tumor Immunology and Microenvironmental Regulation, Guilin Medical University, Guilin, Guangxi 541004, China. ${ }^{2}$ Department of Physiology, Guilin Medical University, Guilin, Guangxi, China. ${ }^{3}$ Department of Breast and Thyroid Surgery, First Affiliated Hospital of Guilin Medical University, Guilin, Guangxi, China. ${ }^{4}$ Department of Pathology and Physiopathology, Guilin Medical University, Guilin, Guangxi, China.
\end{abstract}

Received: 19 November 2017 Accepted: 19 November 2017 Published online: 15 December 2017

\section{Reference}

1. Tian J, Wang Y, Zhang X, Ren Q, et al. Calycosin inhibits the in vitro and in vivo growth of breast cancer cells through WDR7-7-GPR30 Signaling. J Exp Clin Cancer Res. 2017;36(1):153. https://doi.org/10.1186/s13046-0170625-y.

\footnotetext{
* Correspondence: chjian80726@163.com

${ }^{1}$ Key Laboratory of Tumor Immunology and Microenvironmental Regulation, Guilin Medical University, Guilin, Guangxi 541004, China
} 